\title{
First in-human report of the clinical accuracy of thoracolumbar percutaneous pedicle screw placement using augmented reality guidance
}

\author{
Alexander T. Yahanda, MD, MPHS, ${ }^{1}$ Emelia Moore, BS, ${ }^{2}$ Wilson Z. Ray, MD, ${ }^{1}$ \\ Brenton Pennicooke, MD, ${ }^{1}$ Jack W. Jennings, MD, PhD, ${ }^{3}$ and Camilo A. Molina, MD' \\ Departments of ${ }^{1}$ Neurosurgery and ${ }^{3}$ Radiology, Washington University School of Medicine in St. Louis, Missouri; and ${ }^{2}$ Wayne \\ State University School of Medicine, Detroit, Michigan
}

OBJECTIVE Augmented reality $(A R)$ is an emerging technology that has great potential for guiding the safe and accurate placement of spinal hardware, including percutaneous pedicle screws. The goal of this study was to assess the accuracy of 63 percutaneous pedicle screws placed at a single institution using an AR head-mounted display (ARHMD) system.

METHODS Retrospective analyses were performed for 9 patients who underwent thoracic and/or lumbar percutaneous pedicle screw placement guided by ARHMD technology. Clinical accuracy was assessed via the Gertzbein-Robbins scale by the authors and by an independent musculoskeletal radiologist. Thoracic pedicle subanalysis was also performed to assess screw accuracy based on pedicle morphology.

RESULTS Nine patients received thoracic or lumbar AR-guided percutaneous pedicle screws. The mean age at the time of surgery was $71.9 \pm 11.5$ years and the mean number of screws per patient was 7 . Indications for surgery were spinal tumors $(n=4,44.4 \%)$, degenerative disease $(n=3,33.3 \%)$, spinal deformity $(n=1,11.1 \%)$, and a combination of deformity and infection $(n=1,11.1 \%)$. Presenting symptoms were most commonly low-back pain $(n=7,77.8 \%)$ and lower-extremity weakness ( $n=5,55.6 \%$ ), followed by radicular lower-extremity pain, loss of lower-extremity sensation, or incontinence/urinary retention ( $n=3$ each, 33.3\%). In all, 63 screws were placed (32 thoracic, 31 lumbar). The accuracy for these screws was $100 \%$ overall; all screws were Gertzbein-Robbins grade A or B $(96.8 \%$ grade A, $3.2 \%$ grade $B)$. This accuracy was achieved in the thoracic spine regardless of pedicle cancellous bone morphology.

CONCLUSIONS AR-guided surgery demonstrated a 100\% accuracy rate for the insertion of 63 percutaneous pedicle screws in 9 patients (100\% rate of Gertzbein-Robbins grade A or B screw placement). Using an ARHMS system for the placement of percutaneous pedicle screws showed promise, but further validation using a larger cohort of patients across multiple surgeons and institutions will help to determine the true accuracy enabled by this technology.

https://thejns.org/doi/abs/10.3171/2021.5.FOCUS21217

KEYWORDS augmented reality; computer-assisted spine surgery; mixed reality; percutaneous pedicle screw; spine navigation

$\mathrm{A}$ UGMENTED reality (AR) is an emerging technology that has been applied to many different types of surgery, including neurosurgery, orthopedic surgery, genitourinary surgery, and hepatobiliary surgery., Using AR, surgeons can merge virtual interfaces with the physical operative field to provide greater information or navigation capabilities. One of the applications of AR with the highest potential is spinal surgery. ${ }^{3,4}$ AR has been shown previously, both in cadaver studies and in real patients, to be of clinical utility for guiding the placement of spinal hardware. ${ }^{4-8}$ These studies have suggested that pedicle screw placement and tumor resection guided by AR have the potential to be highly accurate and precise. AR thus may be a meaningful surgical adjunct to conventional freehand pedicle screw placement or other spinal procedures and may enable comparable accuracy to robotic pedicle screw placement. ${ }^{3}$ It may also reduce fluoroscopy- or CT-associated radiation exposure to patients and surgeons.

As the applications for AR guidance in spinal surgery increase, more data are required to quantify the utility of this technology. Until this point, little has been written regarding the use of AR guidance for the placement of percutaneous thoracic or lumbar pedicle screws, particularly regarding AR head-mounted display (ARHMD) stereotac- 

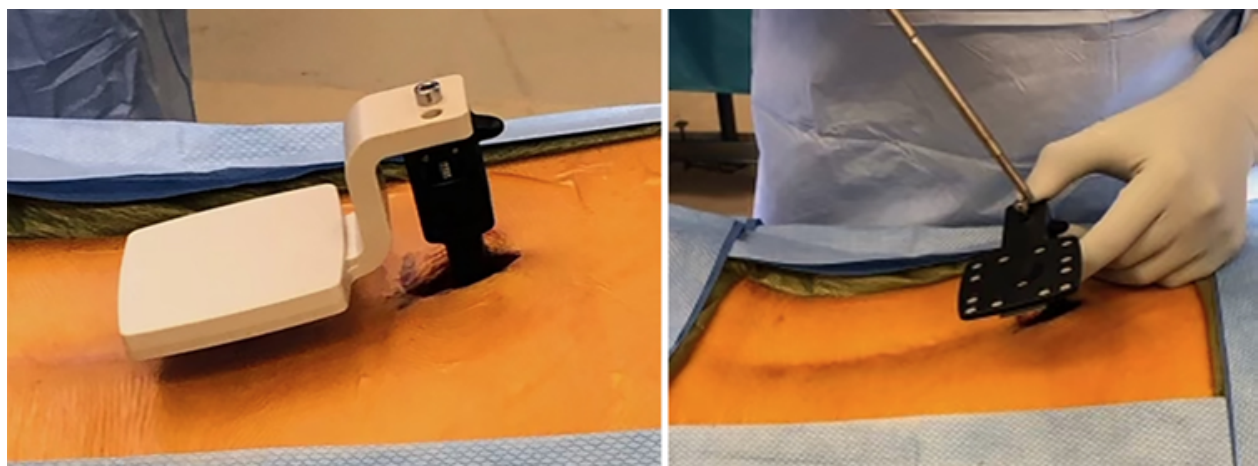

FIG. 1. A registration clamp and marker were placed on spinous processes before intraoperative 3D CT imaging registration (left), after which the registration marker was exchanged for a tracking marker (right).

tic navigation systems. Most studies have centered on different types of navigation, including robotic systems, CTguided neuronavigation, or other modalities. ${ }^{9,10}$ In order to fill this gap in the literature, to our best knowledge, we present the first reported case series of ARHMD-guided thoracolumbar percutaneous pedicle screw placement from a single institution. We assessed the accuracy of pedicle screw placement for 63 screws that were inserted using an FDA-approved ARHMD system.

\section{Methods \\ Data Collection}

This study received institutional review board approval. Cases were retrospectively reviewed to identify patients who underwent thoracic and/or lumbar percutaneous pedicle screw placement guided by an ARHMD system (xvision Spine System, Augmedics). ${ }^{11}$ All cases were performed at a single academic tertiary care center since December 2020. This is a continuous case series, with no cases excluded from analysis from the acquisition of the system to the day of submission of this article. No cases were aborted or converted to open or to another navigation platform. Inclusion criteria were age $>18$ years and placement of percutaneous pedicle screws in the thoracic or lumbar spine. All patients were treated by a single surgeon (C.A.M.). Data extracted from electronic medical records included demographic information (age, sex, and race), prior medical and surgical history, indications for spinal surgery, preoperative symptoms, surgical/instrumentation information (levels of surgery and number of screws placed), pre- and postoperative imaging studies, and intra- or postoperative complications. Data were deidentified prior to analysis.

\section{Surgical Technique}

Patients were prepped and draped in the standard sterile fashion after being positioned on a Jackson table in the prone position. Intraoperative neuromonitoring was used for all patients. Based on the exposure and instrumentation levels, a registration clamp and marker (Fig. 1 left) were placed on spinous processes, and intraoperative 3D CT imaging registration was conducted using an $\mathrm{O}$-arm (Medtronic). The registration marker was then exchanged for a tracking marker (Fig. 1 right), which was placed on the contralateral working side. The surgeons then had the ARHMD device fitted on their heads. The ARHMD provides 3D overlay of the patient's anatomy in a 1:1 size ratio and 2D axial and parasagittal tool trajectory projections (Fig. 2).

The initial entry point was chosen on the 3D projection as if performing freehand instrumentation, and a $2-\mathrm{cm}$ incision was made overlying the entry point of each screw. Using monopolar cautery, the facia was transgressed in a linear fashion. Using a calibrated AR-tracked high-speed drill with an extended 3-mm matchstick burr, a cortical purchase was made in the desired screw trajectory and the trajectory was saved (Fig. 3A). A calibrated AR-tracked 4.35-mm or 5-mm tap on power ream setting (Series 7, Stryker) was used to completely cannulate the desired trajectory (Fig. 3B). A calibrated AR-tracked power screwdriver was then used to drive the screw into final position, and the position was saved on the digital projections (Fig. 3C). All tool exchanges during cortical purchase, tapping, and instrumentation placement were performed without

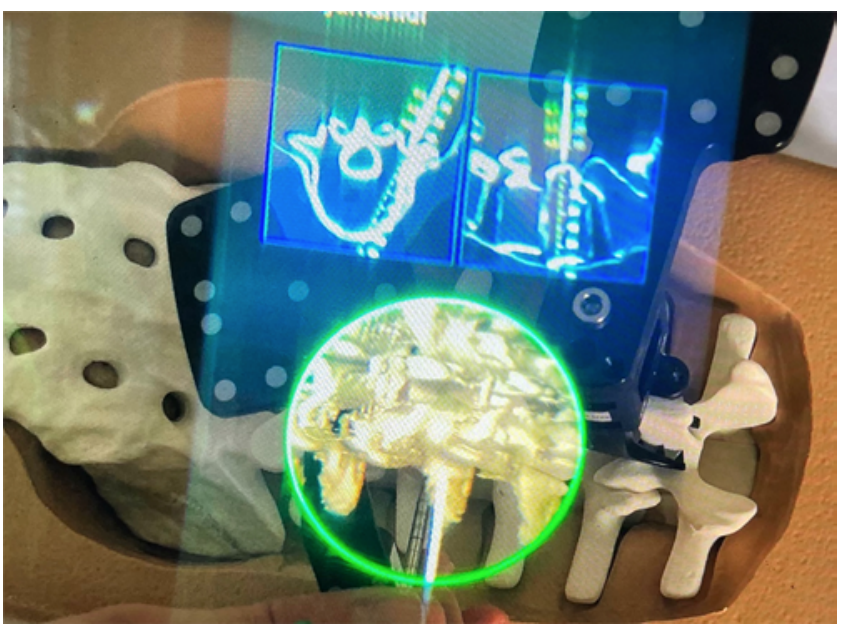

FIG. 2. Example view of the heads-up display after the surgeon had the ARHMD device fitted on their head. The ARHMD provides a 3D overlay of the patient's anatomy in a 1:1 size ratio with $2 \mathrm{D}$ axial and parasagittal tool trajectory projections. 
Yahanda et al.

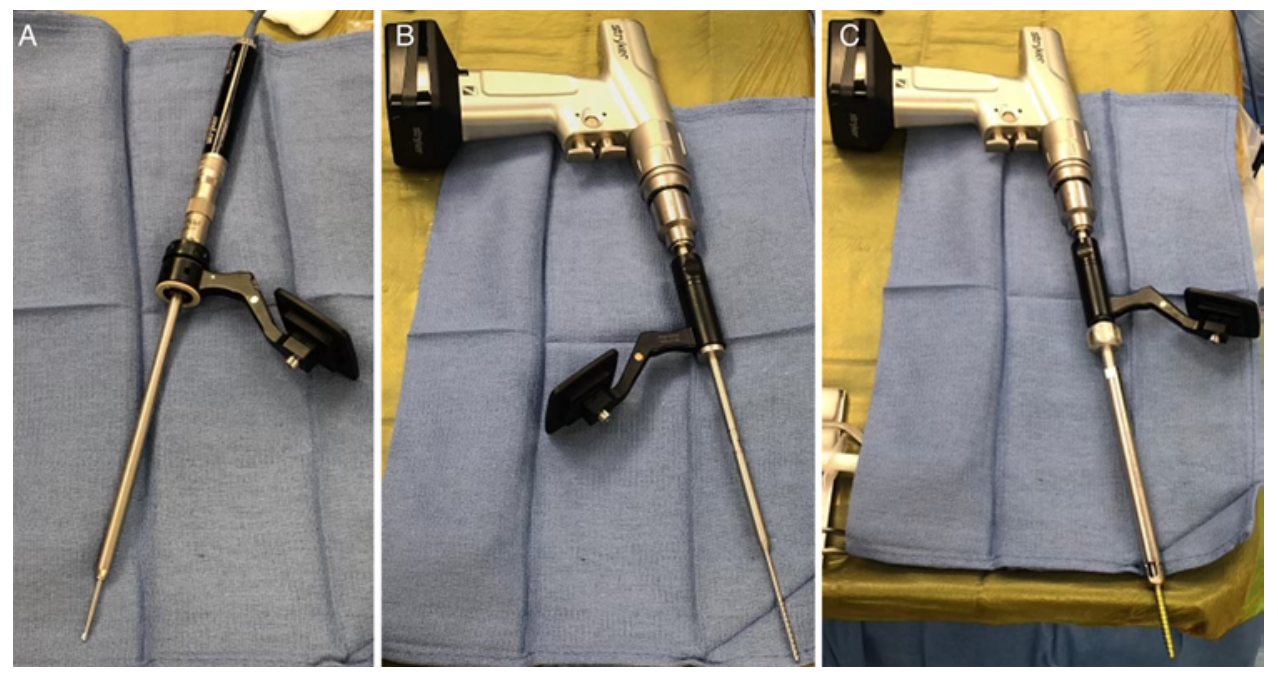

FIG. 3. A: After an incision was made over the entry point for each screw, a calibrated AR-tracked high-speed drill with an extended 3-mm matchstick burr was used to achieve cortical purchase in the desired screw trajectory. B: A calibrated AR-tracked 4.35-mm or 5-mm tap on power ream setting was used to cannulate the desired trajectory. C: A calibrated AR-tracked power screwdriver was used to drive the screw into final position.

K-wires via the use of trajectory projections (Fig. 4) to percutaneously guide the tool exchanges.

Following completion of instrumentation placement, the pedicle marker and registration clamp were removed, after which the remainder of the procedure was completed using standard techniques and tools. Effort was made to place the spinous process clamp over the area that needed a midline opening for further work. Screw placement accuracy was confirmed using either an intraoperative $\mathrm{O}$ arm CT scan or a conventional postoperative CT scan. Of note, the interspinous clip that is required for registration may become obscured or dislodged, resulting in a loss of registrational precision. However, this did not occur in any cases. Frequent anatomical landmark checks were used to confirm preserved registrational accuracy throughout the procedure.

\section{Assessment of Screw Accuracy}

The Gertzbein-Robbins scale ${ }^{12}$ was used to assess the accuracy of pedicle screw placement. The scale was de-
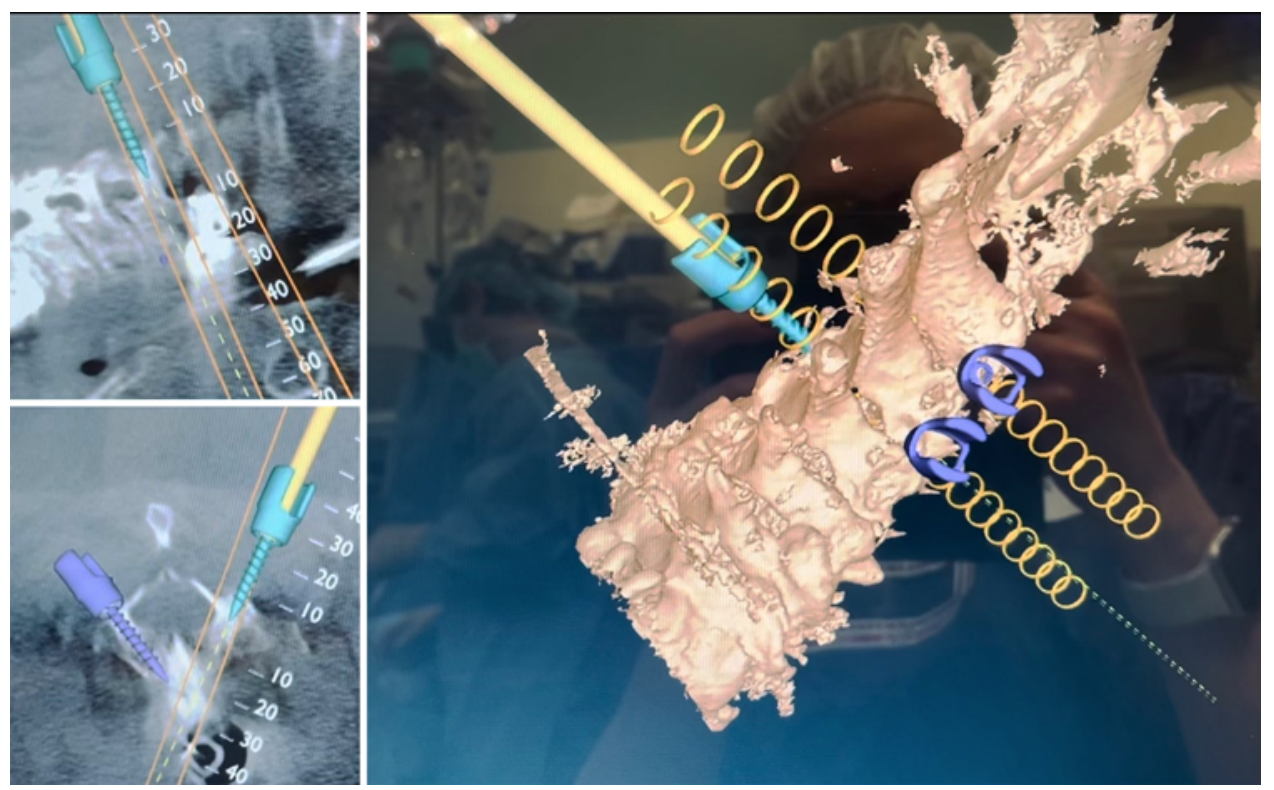

FIG. 4. An example of trajectory projection images that were used to percutaneously guide tool exchanges during surgery. Due to these projection images, all tool exchanges during cortical purchase, tapping, and instrumentation placement were performed without K-wires. 
lineated in the standard grade A to E fashion as follows: grade A (perfect intrapedicular screw localization with no cortical breach), grade B $(<2$-mm cortical breach), grade $\mathrm{C}(<4-\mathrm{mm}$ cortical breach), grade $\mathrm{D}(<6-\mathrm{mm}$ cortical breach), and grade E ( $\geq 6$-mm cortical breach). ${ }^{12}$ Screw accuracy was determined from either postoperative CT images or intraoperative $\mathrm{O}$-arm CT images that had been obtained after screw placement. All images were analyzed via 3-planar assessment at all relevant vertebral levels. Clinical accuracy was measured by two authors and verified by an independent musculoskeletal radiologist. Adequate accuracy of pedicle screw placement was considered to be Gertzbein-Robbins grades A and B.

A further subanalysis of thoracic pedicle screw accuracy was conducted using the pedicle channel classification put forth by Watanabe et al..$^{13}$ Assessment of pedicle morphology was performed using preoperative CT with the gantry placed parallel to the pedicles. Pedicles were classified as type $\mathrm{A}$ to $\mathrm{D}$, where type $\mathrm{A}$ signified a thoracic pedicle with a large cancellous channel, type B signified a small cancellous channel pedicle, type $\mathrm{C}$ signified a cortical channel pedicle (no cancellous bone at the isthmus), and type D signified a slit/absent channel pedicle (no cancellous bone channel, which necessitated juxtapedicular screw placement). The number of pedicles adhering to each classification was recorded, and the accuracy of percutaneous pedicle screw placement was recorded for each classification.

\section{Results}

\section{Patient Characteristics}

Nine patients underwent thoracic and/or lumbar percutaneous pedicle screw placement using the ARHMD system (Table 1). Sixty-three pedicle screws were placed among all patients. Four patients (44.4\%) were female and all were White. The mean age was $71.91 \pm 11.5$ years $( \pm$ $\mathrm{SD}$ ), and the mean BMI was $27.3 \pm 5.6 \mathrm{~kg} / \mathrm{m}^{2}$. Four patients (44.4\%) had a history of prior spinal surgery, all of which were prior decompression and fusion. Regarding medical comorbidities, 5 patients $(55.6 \%)$ had a history of cancer, of which 4 patients had metastatic disease to the spine at the time of their AR-guided surgery. The indications for surgery were degenerative disease in 3 patients (33.3\%), spinal tumor in 4 patients $(44.4 \%)$, spinal deformity in 1 patient $(11.1 \%)$, and a combination of deformity and infection in 1 patient (11.1\%). Presenting symptoms were most commonly low-back pain (7 patients, $77.8 \%$ ) or lower-extremity weakness (5 patients, $55.6 \%$ ), followed by radicular lower-extremity pain, lower-extremity diminished sensation, or incontinence/urinary retention (3 patients each, 33.3\%).

\section{Screw Placement}

A total of 63 percutaneous pedicle screws were placed with ARHMD guidance (Table 2). The mean number of screws per patient was 7 (range 2-14). Thirty-two screws (50.8\%) were placed in the thoracic spine, and 31 (49.2\%) were placed in the lumbar spine. All pedicle screws were placed bilaterally with the exception of 1 lumbar pedicle screw, which could not be placed, as its trajectory was im-
TABLE 1. Patient demographic and clinical information

\begin{tabular}{lc}
\hline \multicolumn{1}{c}{ Variable } & Value \\
\hline Female sex & $4(44.4)$ \\
\hline White race & $9(100)$ \\
\hline Mean age, yrs & $71.91 \pm 11.5$ \\
\hline Mean BMI, kg/m & $27.3 \pm 5.6$ \\
\hline Prior spine surgery & $4(44.4)$ \\
\hline Decompression alone & $0(0.0)$ \\
\hline Decompression \& fusion & $4(44.4)$ \\
\hline Medical comorbidities & \\
\hline Cancer & $5(55.6)$ \\
\hline Osteoporosis & $1(11.1)$ \\
\hline Hypertension & $5(55.6)$ \\
\hline Diabetes & $3(33.3)$ \\
\hline Epidural abscess & $1(11.1)$ \\
\hline Presenting symptoms & $7(77.8)$ \\
\hline Low-back pain & $3(33.3)$ \\
\hline Radicular lower-extremity pain & $5(55.6)$ \\
\hline Lower-extremity weakness & $3(33.3)$ \\
\hline Loss of lower-extremity sensation & $3(33.3)$ \\
\hline Incontinence/urinary retention & $3(33.3)$ \\
\hline Indication for surgery & $2(22.2)$ \\
\hline Degenerative & $4(44.4)$ \\
\hline Deformity* & $1(11.1)$ \\
\hline Tumor &
\end{tabular}

Values represent the number of patients (\%) or mean \pm SD.

* One patient received surgery for a combination of deformity and infection.

peded by prior lateral plate screws at the same level. The accuracy of screw placement was $100 \%$ overall $(100 \%$ for both thoracic and lumbar screws). Of the 63 screws placed, $61(96.8 \%)$ were Gertzbein-Robbins grade A, signifying no cortical breach. There were 2 screws (3.2\%) that were Gertzbein-Robbins grade B, both of which were placed in the thoracic spine in the same patient. No screws were Gertzbein-Robbins grades C to E.

Based on preoperative CT studies, thoracic pedicle subanalyses using the Watanabe classification indicated that $19(59.4 \%)$ of 32 pedicles were type A, 9 (28.1\%) of 32 pedicles were type B, $4(12.5 \%)$ of 32 pedicles were type $\mathrm{C}$, and $0(0.0 \%)$ of 32 pedicles were type $\mathrm{D}$ (Table 3 ). The accuracy of pedicle screw placement was $100 \%$ for type $\mathrm{A}, \mathrm{B}$, and $\mathrm{C}$ pedicles.

\section{Postoperative Outcomes}

There were no intraoperative complications. Postoperatively, all patients reported symptomatic improvement, and there were no postoperative complications at the time of hospital discharge. At the most recent follow-up, all patients reported continued symptomatic improvement. One patient died several weeks after surgery, but this was from their underlying metastatic cancer rather than from their spinal disease or operation. 
TABLE 2. Spinal surgical procedures for each patient, including the number and location of percutaneous pedicle screws

\begin{tabular}{|c|c|c|}
\hline Patient No. & Surgical Procedure & $\begin{array}{l}\text { No. \& Location of Pedicle } \\
\text { Screws Placed }\end{array}$ \\
\hline 1 & $\begin{array}{l}\text { T12 corpectomy for tumor resection; bilat percutaneous pedicle screw placement at T10-11 \& L1-2; } \\
\text { posterior rod fixation from T10 to L2 }\end{array}$ & 4 thoracic, 4 lumbar \\
\hline 2 & $\begin{array}{l}\text { T12-L1 corpectomy followed by bilat percutaneous pedicle screw placement at T10-11 \& L3; unilat } \\
\text { percutaneous pedicle screw placement at L2; posterior rod fixation from T10 to L3 }\end{array}$ & 4 thoracic, 3 lumbar* \\
\hline 3 & $\begin{array}{l}\text { T9-10 hemicorpectomy for tumor resection; bilat percutaneous pedicle screw placement at T7-8 \& } \\
\text { T11-12; posterior rod fixation from T7 to T12 }\end{array}$ & 8 thoracic \\
\hline 4 & Bilat percutaneous pedicle screw placement at C7-T1; posterior rod fixation at C7-T1 & 2 thoracic \\
\hline 5 & $\begin{array}{l}\text { Epidural tumor resection at } S 1-2 \text {; bilat percutaneous pedicle screw placement at L4-5; L4-pelvis } \\
\text { posterior rod fixation }\end{array}$ & 4 lumbar \\
\hline 6 & TLIF at L3-4; bilat percutaneous pedicle screw placement at L3-4; posterior rod fixation at L3-4 & 4 lumbar \\
\hline 7 & TLIF at L3-4; bilat percutaneous pedicle screw placement at L3-4; posterior rod fixation at L3-4 & 4 lumbar \\
\hline 8 & $\begin{array}{l}\text { T11 corpectomy for tumor and pathologic fracture; bilat percutaneous pedicle screw placement at T7- } \\
\text { T10 \& T12-L2; posterior rod fixation at T7-L2 }\end{array}$ & 10 thoracic, 4 lumbar \\
\hline 9 & $\begin{array}{l}\text { Retroperitoneal } L 1-2 \& L 2-3 \text { anterior column release \& } L 1-3 \text { lateral interbody fusion; bilat pedicle screw } \\
\text { placement at T11-L4; posterior rod fixation at T11-L4 }\end{array}$ & 4 thoracic, 8 lumbar \\
\hline
\end{tabular}

TLIF = transforaminal lumbar interbody fusion.

* One patient received unilateral pedicle screw placement at L2 due to interference from prior lateral instrumentation at the same level.

\section{Clinical Case Example}

A 73-year-old male presented with severe back pain, radicular lower-extremity pain, and difficulty walking. The patient had a history of urothelial carcinoma, and a T12L1 Mycobacterium bovis epidural abscess and osteomyelitis for which he had received prior L1-4 laminectomies.

\section{TABLE 3. Percutaneous pedicle screw accuracy and anatomical} classifications

\begin{tabular}{cc}
\hline & Value \\
\hline Total no. of screws & 63 \\
\hline Thoracic & $32(50.8)$ \\
\hline Lumbar & $31(49.2)$ \\
\hline Mean no. of screws per patient & 7 \\
\hline Overall accuracy $(\%)$ & 100 \\
\hline Thoracic & 100 \\
\hline Lumbar & 100 \\
\hline Gertzbein-Robbins grade & \\
\hline A & $61(96.8)$ \\
\hline B & $2(3.2)$ \\
\hline C & $0(0)$ \\
\hline D & $0(0)$ \\
\hline E & $0(0)$ \\
\hline Watanabe classification type $(\mathrm{n}=32)$ & \\
\hline A & $19(59.4)$ \\
\hline B & $9(28.1)$ \\
\hline C & $4(12.5)$ \\
\hline D & $0(0.0)$ \\
\hline
\end{tabular}

Values represent the number of screws (\%).
He presented to our institution for further management of his symptoms. Preoperative CT demonstrated progressive destruction of the T12 and L1 vertebral bodies secondary to his prior osteomyelitis with increased spinal canal stenosis from T12 to L3 and a severe focal kyphotic deformity at T12-L1.

The patient was treated with a 2 -stage operative procedure. For the first stage, he received T12 and L1 corpectomies with anterior arthrodesis from T11 to L2 using an intervertebral cage from T11 to L2 with lateral instrumented fusion. For the second stage, the ARHMD system was used for placement of percutaneous pedicle screws at T10-11 and L3. A unilateral percutaneous pedicle screw was placed at L2; the contralateral L2 pedicle screw could not be placed as it was blocked by the instrumentation from the prior lateral fusion. After successful placement of the percutaneous screws, the patient received posterior rod fixation from T10 to L3.. Both intraoperative O-arm CT and postoperative CT studies demonstrated 100\% Gertzbein-Robbins grade A accuracy for the pedicle screws.

Postoperatively, the patient reported improvements in pain and lower-extremity function. Per the advice of the infectious disease team, he was discharged on rifampin, isoniazid, and pyridoxine. At the most recent follow-up, he still demonstrated muscle weakness in his distal lower extremities but denied back or lower-extremity pain and had regained the ability to ambulate with a walker (Fig. 5).

\section{Discussion}

This is the first reported clinical case series of thoracolumbar ARHMD-guided percutaneous pedicle screw placement from a single institution. We reported an accuracy of $100 \%$ for screw placement in both the thoracic and lumbar spine, regardless of the indications for surgery (including a $96.8 \%$ rate of Gertzbein-Robbins grade A accu- 

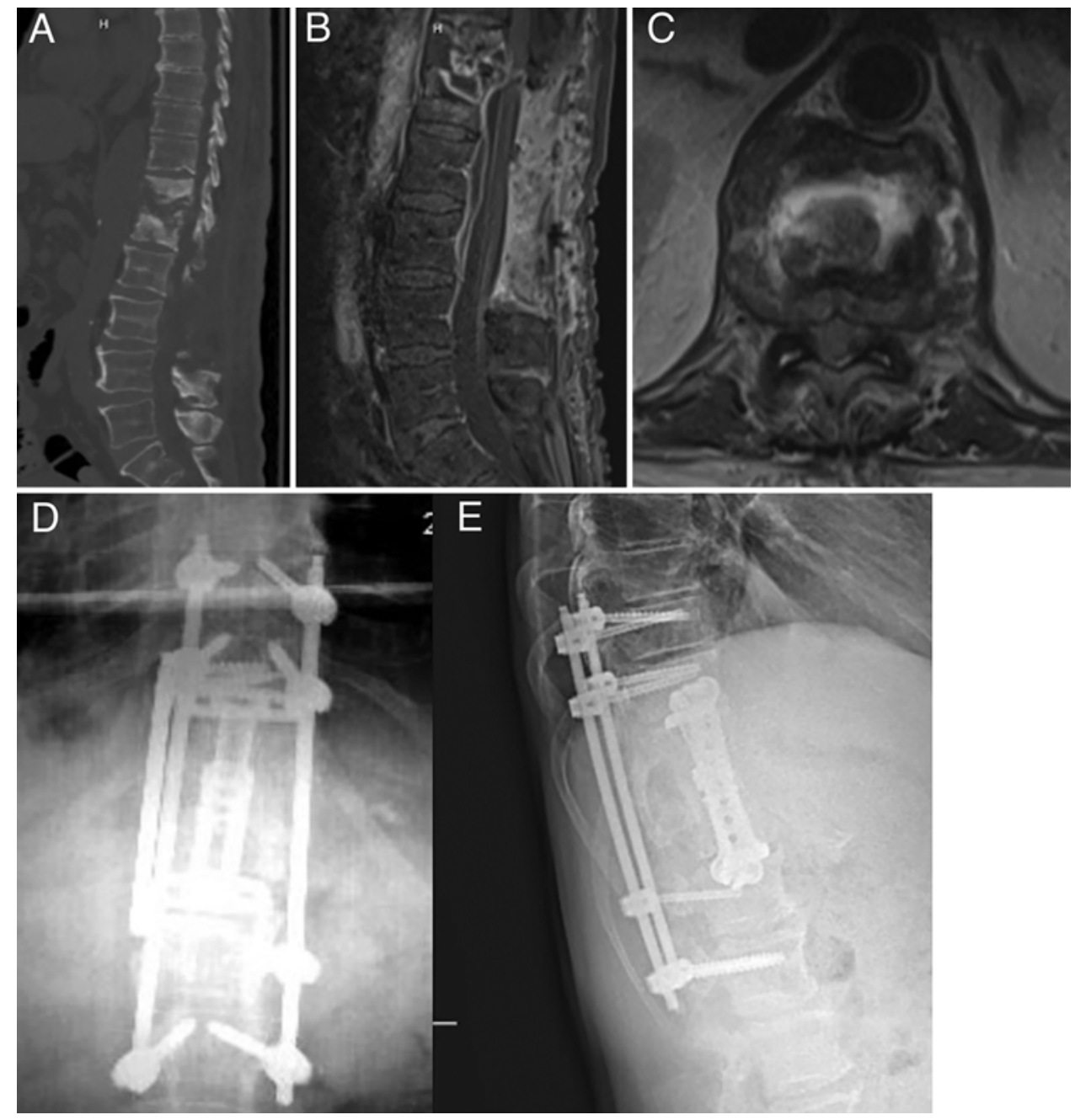

FIG. 5. Images obtained in a 73-year-old man who presented with severe back pain. A-C: Preoperative sagittal CT (A) and sagittal $(B)$ and axial $(C)$ MR images demonstrating destruction of the T12 and L1 vertebral bodies secondary to prior osteomyelitis with increased spinal canal stenosis from T12 to L3 and a severe focal kyphotic deformity at T12-L1. D and E: Postoperative anteroposterior (D) and lateral (E) radiographs demonstrating successful T12 and L1 corpectomies, anterior arthrodesis from T11 to L2 using an intervertebral cage from T11 to L2 with lateral instrumented fusion, and percutaneous pedicle screws with posterior rod fixation from T10 to L3.

racy). Moreover, this $100 \%$ accuracy was obtained in the thoracic spine despite variation in pedicle morphology, as Gertzbein-Robbins grade A or B accuracy was obtained in pedicles that had large, small, or absent cancellous channels (types A-C per the Watanabe classification). Our data contribute to the literature supporting ARHMD systems as a viable means to improve the accuracy of percutaneous pedicle screw placement.

Our results using the ARHMD system are similar to those for other technological adjuncts for pedicle screw placement-most notably, the use of robotic systems. ${ }^{14}$ The first robotic system designed for placement of screws was the SpineAssist robot, which initially demonstrated Gertzbein-Robbins grade A or B accuracy of $97.9 \%$ for 487 screws placed in 112 patients. ${ }^{15}$ Subsequent improvements in the SpineAssist platform have yielded accuracy rates of up to $99 \% .{ }^{14,16}$ Another robotic system, the ExcelsiusGPS, has enabled Gertzbein-Robbins grade A or B accuracy of between $96.6 \%$ and $100 \%$ across multiple studies. ${ }^{16-19}$ Other robotic platforms (ROSA or TianJi) have shown similar accuracy ranging from $97 \%$ to $98.7 \%$ when compared with freehand screw placement. ${ }^{14,20}$ Although we analyzed a somewhat limited patient cohort, our data show that using an ARHMD system for percutaneous pedicle screw placement can provide similar-or superior-accuracy compared with the most commonly used robotic systems. Future studies are necessary to compare AR and robotics on a larger scale, as well as to delineate the differences in clinical workflow, cost savings, and precision between these technologies.

The accuracy of ARHMD-guided pedicle screw placement has been investigated and validated by a small number of prior studies. Over the course of the development of the ARHMD system, it has been shown to enable accuracy between $94.6 \%$ and $99.1 \%$ for lumbosacral and thoracic pedicle screw placement in cadavers as assessed by 
the Gertzbein-Robbins scale. ${ }^{5,6}$ However, in patients, the same ARHMD system enabled $100 \%$ accuracy for pedicle screw placement, although this was only for 6 screws placed in 1 patient. ${ }^{7}$ It also was shown to provide excellent clinical accuracy for osteotomies, discectomies, and pedicle screw placement during posterior en bloc L1 corpectomy for a chordoma. ${ }^{8}$ A different AR system was used for percutaneous pedicle screw placement during transforaminal lumbar interbody fusion with $94 \%$ accuracy; however, this system relies on remote displays and does not use a head-mounted AR display. ${ }^{21}$ Other studies have suggested that AR may provide favorable accuracy compared with fluoroscopy-guided screw placement. ${ }^{22-25}$ Many of these studies utilized the Gertzbein-Robbins scale to assess accuracy of pedicle screws, which allows for direct comparison between our results and this literature. However, using the classification system created by Watanabe et al. ${ }^{13}$ is less common. In our cohort of patients, only $12.5 \%$ of thoracic pedicles were type $\mathrm{C}$ and none were type $\mathrm{D}$ based on preoperative CT images, which indicated that most patients had relatively accessible cancellous pedicle channels. Additional studies examining patients with type $\mathrm{C}$ or $\mathrm{D}$ pedicle channels would be useful to discern the ability of AR to accurately guide screw placement given the most challenging thoracic pedicle morphology.

Our $100 \%$ Gertzbein-Robbins grade A or B rate was achieved by a single surgeon who is very familiar with the ARHMD system. Therefore, this accuracy rate may not be obtained at first by all surgeons who are using ARHMD technology for the placement of percutaneous pedicle screws, although it represents the accuracy that may ultimately be achieved. The ARHMD interface may present initial difficulties due to visual discomfort from having to adapt to the context of AR. The heads-up display presented by the ARHMD headset may prove disorienting for surgeons upon first use. Operators will no doubt need time to become comfortable with having a virtual interface overlaid on top of the patient and operative field. However, as with any new surgical technology, the ARHMD should become more easily incorporated into the surgical workflow with continued use. Finally, as with any navigation system, use of this technology may not be feasible in a subset of patients. Intraoperative CT scans are necessary for registration, so situations in which these cannot be obtained (for patients with too large a body habitus, for instance) will prevent the use of AR.

\section{Limitations}

The primary limitation of this study is its low number of patients $(n=9)$ and pedicle screws $(n=63)$. Although we found a $100 \%$ accuracy rate in placing percutaneous pedicle screws using ARHMD guidance, studies on this topic using a greater number of patients are necessary to discern the true accuracy enabled by this technology. Furthermore, these 9 patient procedures were all performed at a single institution by one surgeon who is familiar with the ARHMD system at the time of the first case. Thus, while our data represent what is achievable using AR in the hands of an experienced surgeon, they do not reflect what may happen when numerous surgeons with differing experience levels from multiple institutions place percutaneous pedicle screws with AR. The indications for surgery also varied among these 9 patients, which provided few cases per individual type of pathology or surgical procedure. Again, future studies with larger patient cohorts will further delineate the utility of ARHMD guidance for a broader array of surgeries and conditions. Nonetheless, we find that the data are of value given that it is the first in-human report of the clinical performance of this platform in the placement of percutaneous thoracolumbar pedicle screws.

It is possible that assigning Gertzbein-Robbins grades may have been influenced by whether postoperative images were performed using an $\mathrm{O}$-arm or a conventional CT scanner since O-arm image quality is inferior. That said, all of the authors who assigned Gertzbein-Robbins grades (including an experienced radiologist) reported no difficulties assigning the same grades to each patient, indicating that our institution's O-arm image quality was sufficient for these analyses. Our study examined the accuracy of percutaneous pedicle screws guided by ARHMD as assessed via the Gertzbein-Robbins scale, but it did not assess the precision of pedicle screw placement. That is, we did not determine whether these screws were placed exactly where the surgeon intended them to be placed. The precision of this particular ARHMD system has been examined previously in both cadavers and patients, ${ }^{6,7}$ and as our institution's experience with ARHMD-guided surgery increases, we are keenly interested in determining both the accuracy and precision of pedicle screws placed using AR on a larger number of patients and among multiple surgeons.

\section{Conclusions}

We report the first in-human series of patients who underwent percutaneous thoracic and lumbar pedicle screw placement guided by an ARHMD system. AR-guided surgery demonstrated a $100 \%$ accuracy rate for 63 screws placed, including a $96.8 \%$ rate of Gertzbein-Robbins grade A screw placement. Accurate thoracic screw placement was universally achieved irrespective of thoracic pedicle bone morphology (Watanabe types A-C). Using AR for the placement of percutaneous pedicle screws shows promise, but further validation using a larger cohort of patients across a higher number of surgeons and institutions is required to elucidate the true benefits of this technology.

\section{References}

1. Vávra P, Roman J, Zonča P, Ihnát P, Němec M, et al. Recent development of augmented reality in surgery: a review. $J$ Healthc Eng. 2017;2017:4574172.

2. Fida B, Cutolo F, di Franco G, Ferrari M, Ferrari V. Augmented reality in open surgery. Updates Surg. 2018;70(3):389400.

3. Burström G, Persson O, Edström E, Elmi-Terander A. Augmented reality navigation in spine surgery: a systematic review. Acta Neurochir (Wien). 2021;163(3):843-852.

4. Vadalà G, De Salvatore S, Ambrosio L, Russo F, Papalia R, Denaro V. Robotic spine surgery and augmented reality systems: a state of the art. Neurospine. 2020;17(1):88-100.

5. Molina CA, Theodore N, Ahmed AK, Westbroek EM, Mirovsky $\mathrm{Y}$, et al. Augmented reality-assisted pedicle screw insertion: a cadaveric proof-of-concept study. J Neurosurg Spine. 2019;31(1):139-146. 
6. Molina CA, Phillips FM, Colman MW, Ray WZ, Khan M, et al. A cadaveric precision and accuracy analysis of augmented reality-mediated percutaneous pedicle implant insertion. $J$ Neurosurg Spine. 2021;34(2):316-324.

7. Molina CA, Sciubba DM, Greenberg JK, Khan M, Witham T. Clinical accuracy, technical precision, and workflow of the first in human use of an augmented-reality head-mounted display stereotactic navigation system for spine surgery. Oper Neurosurg (Hagerstown). 2021;20(3):300-309.

8. Molina CA, Dibble CF, Lo SFL, Witham T, Sciubba DM. Augmented reality-mediated stereotactic navigation for execution of en bloc lumbar spondylectomy osteotomies. $\mathrm{J} \mathrm{Neu-}$ rosurg Spine. 2021;34(5):700-705.

9. Perdomo-Pantoja A, Ishida W, Zygourakis C, Holmes C, Iyer RR, et al. Accuracy of current techniques for placement of pedicle screws in the spine: a comprehensive systematic review and meta-analysis of 51,161 screws. World Neurosurg. 2019;126:664-678.e3.

10. Meola A, Cutolo F, Carbone M, Cagnazzo F, Ferrari M, Ferrari V. Augmented reality in neurosurgery: a systematic review. Neurosurg Rev. 2017;40(4):537-548.

11. Dibble CF, Molina CA. Device profile of the XVision-spine (XVS) augmented-reality surgical navigation system: overview of its safety and efficacy. Expert Rev Med Devices. 2021;18(1):1-8.

12. Gertzbein SD, Robbins SE. Accuracy of pedicular screw placement in vivo. Spine (Phila Pa 1976). 1990;15(1):11-14.

13. Watanabe K, Lenke LG, Matsumoto M, Harimaya K, Kim YJ, et al. A novel pedicle channel classification describing osseous anatomy: how many thoracic scoliotic pedicles have cancellous channels? Spine (Phila Pa 1976). 2010;35(20): 1836-1842.

14. Joseph JR, Smith BW, Liu X, Park P. Current applications of robotics in spine surgery: a systematic review of the literature. Neurosurg Focus. 2017;42(5):E2.

15. van Dijk JD, van den Ende RPJ, Stramigioli S, Köchling M, Höss N. Clinical pedicle screw accuracy and deviation from planning in robot-guided spine surgery: robot-guided pedicle screw accuracy. Spine (Phila Pa 1976). 2015;40(17):E986E991.

16. Khan A, Meyers JE, Siasios I, Pollina J. Next-generation robotic spine surgery: first report on feasibility, safety, and learning curve. Oper Neurosurg (Hagerstown). 2019;17(1): 61-69.

17. Huntsman KT, Ahrendtsen LA, Riggleman JR, Ledonio CG. Robotic-assisted navigated minimally invasive pedicle screw placement in the first 100 cases at a single institution. J Robot Surg. 2020;14(1):199-203.

18. Jiang B, Pennington Z, Zhu A, Matsoukas S, Ahmed AK, et al. Three-dimensional assessment of robot-assisted pedicle screw placement accuracy and instrumentation reliability based on a preplanned trajectory. J Neurosurg Spine. 2020; 33(4):519-528.

19. Godzik J, Walker CT, Hartman C, de Andrada B, Morgan $\mathrm{CD}$, et al. A quantitative assessment of the accuracy and reliability of robotically guided percutaneous pedicle screw placement: technique and application accuracy. Oper Neurosurg (Hagerstown). 2019;17(4):389-395.
20. Han X, Tian W, Liu Y, Liu B, He D, et al. Safety and accuracy of robot-assisted versus fluoroscopy-assisted pedicle screw insertion in thoracolumbar spinal surgery: a prospective randomized controlled trial. J Neurosurg Spine. 2019; 30(5):615-622.

21. Charles YP, Cazzato RL, Nachabe R, Chatterjea A, Steib JP, Gangi A. Minimally invasive transforaminal lumbar interbody fusion using augmented reality surgical navigation for percutaneous pedicle screw placement. Clin Spine Surg. Published online February 5, 2021. doi:10.1097/ BSD.0000000000001132

22. Yuk FJ, Maragkos GA, Sato K, Steinberger J. Current innovation in virtual and augmented reality in spine surgery. Ann Transl Med. 2021;9(1):94.

23. Liebmann F, Roner S, von Atzigen M, Scaramuzza D, Sutter $\mathrm{R}$, et al. Pedicle screw navigation using surface digitization on the Microsoft HoloLens. Int J CARS. 2019;14(7):11571165.

24. Ma L, Zhao Z, Chen F, Zhang B, Fu L, Liao H. Augmented reality surgical navigation with ultrasound-assisted registration for pedicle screw placement: a pilot study. Int J CARS. 2017;12(12):2205-2215.

25. Elmi-Terander A, Burström G, Nachabe R, Skulason H, Pedersen $\mathrm{K}$, et al. Pedicle screw placement using augmented reality surgical navigation with intraoperative 3D imaging: a first in-human prospective cohort study. Spine (Phila Pa 1976). 2019;44(7):517-525.

\section{Disclosures}

Dr. Ray: royalties from Acera and consultant for DePuy Synthes, NuVasive, and Globus. Dr. Jennings: consultant for Stryker, Bard/ $\mathrm{BD}$, Agnovos, Varian, and Boston Scientific. Dr. Molina: consultant for and stock options in Augmentics.

\section{Author Contributions}

Conception and design: Molina, Ray, Pennicooke. Acquisition of data: Molina, Yahanda. Analysis and interpretation of data: Molina, Yahanda, Jennings. Drafting the article: all authors. Critically revising the article: all authors. Reviewed submitted version of manuscript: all authors. Approved the final version of the manuscript on behalf of all authors: Molina. Statistical analysis: Yahanda. Administrative/technical/material support: Molina, Ray, Pennicooke, Jennings. Study supervision: Molina.

\section{Supplemental Information Videos}

Video Abstract. https://vimeo.com/572552952.

\section{Correspondence}

Camilo A. Molina: Washington University School of Medicine, St. Louis, MO.cmolina@wustl.edu. 